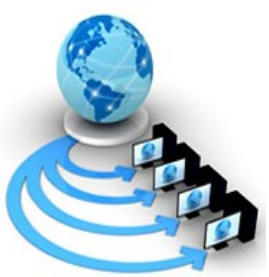

Volume 9, No. 5, September-October 2018

\title{
PERFORMANCE ANALYSIS OF ROUTING ALGORITHMS IN MESH TOPOLOGY
}

\author{
Dr. A .Banumathi, \\ Department Of Computer Science, \\ Government Arts College Karur,India
}

\author{
N.Savithiri \\ Department Of Computer Science, \\ Government Arts College Karur,India
}

\begin{abstract}
This work implements an improved XY routing protocol for analyzing the performance of wireless mesh topology. The wireless mesh topology has become a popular interconnection architecture for constructing massively parallel multi-processors. The examined partially adaptive $\mathrm{XY}$ routing algorithms for different network sizes based on 2-D wireless mesh topology. But it has a routing problem such as highest priority of QUEUE, more number of retransmission and takes delay time to receive the packet. So there is a need for novel system to overcome the drawbacks of deadlock.

The additional implements both odd even turn model and delay tolerant model. It makes message routing very minimal. In additionally, this comparison results in a smaller fluctuation of the network performance with respect to different traffic patterns. In addition, the node failure scenario is also considered so that if the path contains that node, then new alternate path from the failure node to the destination is recalculated or taken from cache if previous path information is stored. The result comparative shows that the Odd-Even Turn XY routing algorithm for best measuring of node throughput, average latency, average extra delay, efficiency, and packet lost and network throughput.
\end{abstract}

Keywords : wireless Mesh network, Mesh Topology, XY routing algorithm, AMMNET

\section{INTRODUCTION}

A network is just a bunch of 2 or additional Personal Computers connected along. Many varieties of networks exist, however the foremost common varieties of networks area unit Local-Area Networks (LANs), and Wide-Area Networks (WANs). In a LAN, computers area unit connected along inside a "local" space (for example, associate in associate workplace or home). In a WAN, computers area unit more apart and area unit connected via telephone/communication lines, radio waves or different means that of affiliation. Networks area unit sometimes classified victimization 3 properties: Topology, Protocol and design.

Topology specifies the geometric arrangement of the network. Common topologies are a bus, ring and star. The examine a figure showing the three common varieties of network topologies here. Protocol specifies a typical set of rules and signals the computers on the network use to speak. Most networks use local area network, however some networks could use IBM's Token Ring protocol. we have a tendency to advocate local area network for each home and workplace networking. For additional info, please choose the local area network link on the left.

Architecture refers to one of the two major varieties of network architecture: Peer-to-peer or client/server. In an exceedingly Peer-to-Peer networking configuration, there's no server, and computers merely connect with one another in an exceedingly workgroup to share files, printers and web access. This is often most typically found in home configurations and is just sensible for workgroups of a dozen or less computers. In associate exceedingly client/server network there's sometimes an NGO Domain Controller, to that all of the computers go surfing. This server will give numerous services, as well as centrally routed web Access, mail (including e-mail), and file sharing and printer access, further as making certain security across the network. This is often most typically found in company configurations, wherever network security is crucial.

The first step in the software development life cycle is the identification of the problem. As the success of the system depends largely on how accurately a problem is identified. The existing system designing the $\mathrm{XY}$ algorithm is twofold. These problems are analysis in this thesis. There is no application with this feature to communicate securely as well as faster. So, this thesis identifies that, if helps to solve the problem through the application.

- Tracks the users and dynamically adapt XY routing the network topology to seamlessly support both their Odd and Even turn XY model and Delay Odd Even XY Routing algorithm.

- Searching for disappearing mobile client communication packet is carried out.

- Minimizing routing paths is considered

There are presently 2 variations of mobile wireless networks infrastructure and Infrastructure less networks. The infrastructure networks, additionally called Cellular network, have mounted and wired gateways. they need mounted base stations that area unit 
connected to different base stations through wires. The transmission vary of a base station constitutes a cell. All the mobile nodes lying inside this cell connects to and communicates with the closest bridge (base station). A hand off happens as mobile host travels out of vary of 1 Base Station and into the vary of another and therefore, mobile host is ready to continue communication seamlessly throughout the network. Example of this sort includes workplace wireless native space networks (WLANS).

\section{LITERATURE REVIEW}

In this paper [1], the extremely thriving design and supporting protocols of today's net operate poorly once baby-faced with operational environments characterized by terribly long delay methods and frequent network partitions. These issues are exacerbated by finish nodes that have severe power or memory constraints. usually deployed in mobile and extreme environments lacking "always-on" infrastructure, several such networks have their own specialised protocols, and don't utilize scientific discipline. to realize ability between them, they propose a spec and application interface structured around optionally-reliable asynchronous message forwarding, with restricted expectations of end-to-end property and node resources. The design operates as AN overlay higher than the transport layers of the networks it interconnects, and provides key services like in-network knowledge storage and retransmission, practical naming, attested forwarding and a coarse-grained category of service.The architecture operates as an overlay above the transport layers of the networks it interconnects, and provides key services such as in-network data storage and retransmission, interoperable naming, authenticated forwarding and a coarse-grained class of service

In this paper [2], Analysis and modeling of wireless networks greatly depend upon understanding the structure of underlying mobile nodes. During this paper they gift 2 clump algorithms to work out the amount of teams and their identities: k-means chain and spectral clump. Totally different from ancient k-means clump, k-means chain will determine the amount of teams in dynamic graphs, and therefore the chaining method may keep track of cluster trajectories over the complete trace. The second approach uses spectral clump that measures the similarities between every node try to cluster nodes of comparable behaviors. They show that important data of a quality trace, like the amount of teams and cluster members, is exactly extracted with very little or no previous information of the properties of a trace.

In this paper [3], within the performance analysis of a protocol for a billboard hoc network, the protocol ought to be tested below realistic conditions together with, however not restricted to, a wise transmission vary, restricted buffer house for the storage of messages, representative information traffic models, and realistic movements of the mobile users (i.e., a quality model). This paper could be a survey of quality models that area unit utilized in the simulations of accidental networks. They describe many quality models that represent mobile nodes whose movements area unit freelance of every different (i.e., entity quality models) and a number of other quality models that represent mobile nodes whose movements area unit smitten by one another (i.e., cluster quality models). The goal of this paper is to gift variety of quality models so as to supply researchers a lot of informed selections after they area unit deciding upon a quality model to use in their performance evaluations. Lastly, they gift simulation results that illustrate the importance of selecting a quality model within the simulation of a billboard hoc network protocol. Specifically, they illustrate however the performance results of a billboard hoc network protocol drastically modification as a results of dynamical the quality model simulated.

In this paper [5], they gift a survey of assorted quality models in each cellular networks and multi-hop networks. They show that cluster motion happens oft in accidental networks, and introduce a completely unique cluster quality model - indicator cluster quality (RPGM) - to represent the connection among mobile hosts. RPGM are often pronto applied to several existing applications. Moreover, by correct selection of parameters, RPGM are often wont to model many quality models that were antecedently projected. one in all the most themes of this paper is to research the impact of the quality model on the performance of a selected network protocol or application.

RPGM model is implementing to two completely different network protocol eventualities, clump and routing, and have evaluated network performance below completely different quality patterns and for various protocol implementations. needless to say, the results indicate that completely different quality patterns have an effect on the assorted protocols in several ways in which. Especially, the ranking of routing algorithms is influenced by the selection of quality pattern. One of the main themes of this paper is to investigate the impact of the mobility model on the performance of a specific network protocol or application

In this paper [6], Realistic models for node movement are essential in simulating mobile ad hoc networks. Many MANET scenarios are most realistically represented using group movement, but existing group movement models depict individual group members as independent actors moving randomly. For many scenarios however, group movement implies a common goal or orientation, and hence an inherent structure to the group. They show that this structure can be defined a-priori, and that knowledge of it will result in more accurate simulations. This paper presents the Structured Group Mobility Model (SGMM), which parameterizes group structure and generates movement sequences for use in simulations. They define the model and demonstrate how such a model of node mobility may be used in creating simulations of several MANET scenarios. They compare simulations using the SGMM with other models using various routing algorithms. Our preliminary results indicate that accurate representation of group structure has a significant effect on the overall simulation, particularly in the area of link stability. The results also imply the need for routing algorithms that take group structure into account. 
In this paper [10], Realistic models for node movement area unit essential in simulating mobile accidental networks. several Edouard Manet eventualities area unit most realistically diagrammatic victimisation cluster movement, however existing cluster movement models depict individual cluster members as freelance actors moving at random. for several eventualities but, cluster movement implies a standard goal or orientation, ANd thence an inherent structure to the cluster. They show that this structure are often outlined apriori, which information of it'll end in a lot of correct simulations. This paper presents the structured cluster quality Model (SGMM), that parameterizes cluster structure and generates movement sequences to be used in simulations. They outline the model and demonstrate however such a model of node quality could also be utilized in making simulations of many Autonomous MANET eventualities. They compare simulations victimisation the SGMM with different models victimisation varied routing algorithms. Our preliminary results indicate that correct illustration of cluster structure includes a vital impact on the general simulation, significantly within the space of link stability. The results additionally imply the necessity for routing algorithms that take cluster structure under consideration.

\section{ROUTING TECHNIQUES}

The existing system bestowed the model for coming up with part reconciling hollow routing algorithms. That theme provides a lot of even routing adaptiveness and demonstrates that communication performance of the meshes could also be improved beneath non-uniform traffics victimisation the XY base odd even flip model.

The XY base odd even flip model presents a model for coming up with reconciling hollow routing algorithms for meshes. The model restricts the locations wherever some turns will be taken in order that stalemate is avoided. as compared with previous strategies (XY routing method), the degree of routing adaptiveness provided by the model is a lot of even for various source-destination pairs. The wireless mesh network might get pleasure from this feature in terms of communication potency.

The odd-even flip model makes message routing terribly minimal . additionally, this property ends up in a smaller fluctuation of the network performance with relevancy completely different traffic patterns. additionally, the node failure state of affairs is additionally thought-about in order that if the trail contains that node, then new alternate path from the failure node to the destination is recalculated or taken from cache if previous path info is keep.

\section{A) XY ROUTING ALGORITHM}

Wang Zhang and Ligang Hou[13] planned Classic XY(Static $\mathrm{XY}$ OR XY) routing rule that is one quite distributed settled routing algorithms .XY routing is one among the kind of Dimension order routing (DOR) that could be a generally a borderline flip rule and is additional appropriate for networks victimisation mesh or torus topology. XY routing rule routes packets initial in $\mathrm{x}$-direction (or horizontal direction) to the proper column and so in $\mathrm{y}$ - direction (or vertical direction) to the receiver. In $\mathrm{XY}$ routing are the addresses of the routers square measure their XY-coordinates. One among the benefits of $X Y$ routing is that it ne'er runs into impasse or livelock [8]. Figure II. XY routing from router A to router B in $4 \times 4$ secret agents but despite the benefits of simplified calculation and removal of impasse or livelock downside, there square measure some issues within the ancient $\mathrm{XY}$ routing.

One necessary issue with ancient $\mathrm{XY}$ routing rule is that the rule causes the most important load within the middle of the network that doesn't extend the traffic frequently over the complete network. so there's a desire for algorithms that equalize the traffic load over the complete network and square measure nonetheless easy and economical.



Fig.3.1 Routing Algoritham

XY routing could be a dimension order routing that routes packets initial in $\mathrm{X}$ - or horizontal direction to the right column and so in $y$ - or vertical direction to the receiver. XY routing suits well on a network victimization mesh or torus topology. Addresses of the routers area unit their XY-coordinates. XY routing ne'er runs into dead end or livelock. [15]

There are some issues within the ancient XY routing. The traffic doesn't extend frequently over the complete network as a result of the algorithmic program causes the largest load within the middle of the network. there's a requirement for algorithms that equalize the traffic load over the complete network

Inputs: Coordinates of source node ( $X$ sour ce, $Y$ sour ce), destination node ( $X$ dest, $Y$ dest),

2: Output: Selected output Channel

3: Begin

4: Xoffset $:=$ Xdest-Xsource; Yoffset $:=$ Ydest-Ysource;

5: if $($ Xoffset $=0)$ and $($ Yoffset $=0)$ then

6: return

7: else

8: if (Yoffset $>0)$ then

9: return $Y+$;

10: end if

11: if $($ Yoffset $<0)$ then

12: return $Y-$;

13: if $($ Xoffset $>0)$ then

14: return $X+$;

15: end if

16: if $($ Xoffset $<0)$ then

17: return $X-$;

18: end if 
19: end if

20: end if

\section{ADVANTAGES}

- When a packet keeps spinning around its destination while not ever reaching it, this example is noted as deadlock. it's a typical drawback that exists in nonminimal kind of routing algorithms.

- $\quad$ TTL uses a counter to count however long a packet has cosmopolitan within the network and because the counter reaches some planned threshold, the packet is far-away from the network. Otherwise is to administer packets a priority supported their age.

- The oldest packet perpetually gets the very best priority and can be routed forward.

\section{DISADVANTAGES}

- A stalemate typically happens once 2 or a lot of packets ar looking forward to one another to be routed forward. In such a case of these packets reserve some resources and ar waiting one another to unharness the resources.

- Routers don't unharness the resources before they get the new resources and then the routing is fast.

\section{B) ADAPTIVE XY ROUTING ALGORITHM}

Pseudo adaptational XY routing is works settled or adaptational mode betting on the state of the network. Algorithmic program works in settled mode once the network isn't or solely slightly full. When network becomes blocked, the algorithmic program switches to the adaptive mode and starts to go looking routes that don't seem to be full. Pseudo adaptive XY routing works is a mesh network that consists of routers, wires and IPblocks. Each router has 5 duplex ports: north, south, east, west and native. Native port connects router to its native core whereas the opposite ports are connected to neighboring routers. Every port encompasses a tiny temporary storage buffer and a 2-bit standing symbol known as quantity load worth.

Identifier tells to different routers if the router is full and can't settle for new packets. A router assigns priorities to incoming packets once there are over one coming at the same time. Packets from north have the very best priority, then south, east and finally packets incoming from west have very cheap priority. While a conventional XY routing causes network hundreds a lot of within the middle of the network than to lateral areas, the pseudo adaptational algorithmic program divides the traffic more equally over the full network.

INPUTS: Current router address- Xcurrent is (X-coordinate of Current router) and Ycurrent is ( $\mathrm{Y}$-coordinate of Current router).
Destination router address- Xdestination is (X-coordinate of Destination router) and Ydestination is (Y-coordinate of Destination router).

Procedure: Begin Ex = Xdestination - Xcurrent Ey = Ydestination - Ycurrent If $(\mathrm{Ex}<0)$ then // West directed packet

\section{Select Output Channel $=$ WEST; \\ End if; \\ If $(\mathrm{Ex}>0$ and $\mathrm{Ey}<0)$ then}

Select Output Channel between (EAST, SOUTH); End if;

If $(\mathrm{Ex}>0$ and $\mathrm{Ey}>0)$ then

Select Output Channel between (EAST, NORTH);

End if;

If $(E x>0$ and $E y=0)$ then

Select Output Channel = EAST;

End if;

If $(\mathrm{Ex}=0$ and $\mathrm{Ey}<0)$ then

Select Output Channel = SOUTH;

End if;

If $(E x=0$ and $E y>0)$ then

Select output Channel $=$ NORTH;

End if;

If $(E x=0$ and $E y=0)$ then

Select Output Channel = LOCAL port;

End if;

End.

End if;

If $(\mathrm{Ex}=0$ and $\mathrm{Ey}>0)$ then

Select output Channel $=$ NORTH;

End if;

If $(\mathrm{Ex}=0$ and $\mathrm{Ey}=0)$ then

Select Output Channel = LOCAL port;

End if;

End.

$>$ Adaptive algorithms regularly require more calculations and with a specific end goal to choose the right way they require some data about the present condition of the network.

$>$ Routing and packet forwarding takes place at a time in adaptive algorithms after every hop it selects the following jump as per the present condition of the hubs and connections activity. In this manner there is dependably on the probability of event that occurs.

\section{C) SURROUNDING XY ROUTING}

Surrounding $\mathrm{XY}$ routing $(\mathrm{S}-\mathrm{XY})$ has 3 totally different routing modes. N-XY (Normal XY) mode works rather like the fundamental $\mathrm{XY}$ routing. It routes packets 1st on coordinate axis and so on coordinate axis. Routing stays on NXY mode as long as network isn't blocked and routing doesn't meet inactive routers. SH$\mathrm{XY}$ (Surround horizontal XY) mode is employed once the router's left or right neighbor is deactivated. Correspondly the third mode SV-XY (Surround vertical $\mathrm{XY}$ ) is employed once the higher or lower neigbor of the router is inactive. The SH-XY mode routes packets to 
the right column on the grounds of coordinates of the destination.

The rule bypasses packets round the inactive routers on the shortest doable path. The case could be a bit totally different within the SV-XY mode as a result of the packets area unit already within the right column. Packets are routed to left or right. Operation in SH-XY and SV-XY modes is shown in Figure ten. The routers within the SH-XY and SV-XY modes add a little symbol to the packets that tells to different routers that these packets area unit routed exploitation SH-XY or SV-XY mode. So the opposite routers don't send the packets backwards. Encompassing XY routing is employed in a very dynamic network and this technique supports to communication between modules that are dynamically placed on a tool

\section{ADVANTAGES}

- When a packet keeps spinning around its destination while not ever reaching it, this example is said as deadlock. it's a typical drawback that exists in nonminimal sort of routing algorithms.

- TTL uses a counter to count however long a packet has cosmopolitan within the network and because the counter reaches some preset threshold, the packet is far from the network. In a different way is to grant packets a priority supported their age.

- The oldest packet perpetually gets the very best priority and can be routed forward.

\section{DISADVANTAGES}

- A stalemate typically happens once two or additional packets are watching for one another to be routed forward. In such a case of these packets reserve some resources and area unit waiting one another to unharness the resources.

- Routers don't unharness the resources before they get the new resources then the routing is barred.

\section{D) XY BASE ODD EVEN TURN MODEL}

AMMNET may be a mesh-based infrastructure that forwards knowledge for mobile purchasers as shown in Fig. 3.3. A consumer will connect with any close mesh node that helps relay knowledge to the destination mesh node via multihop forwarding. For easy description, during this paper we tend to use the terms "mesh node" and "router" interchangeably. Like stationary wireless mesh networks, wherever routers are deployed in fastened locations, routers in associate AMMNET will forward knowledge for mobile clients on the routing ways engineered by any existing impromptu routing protocols.

not like stationary wireless mesh networks, wherever routers area unit deployed at fastened locations, routers in associate AMMNET area unit mobile platforms with autonomous movement capability [5]. They're equipped with positioning devices like GPS, to supply steering aid whereas trailing mobile clients. $\triangle$ Mobile mesh node Mobile client

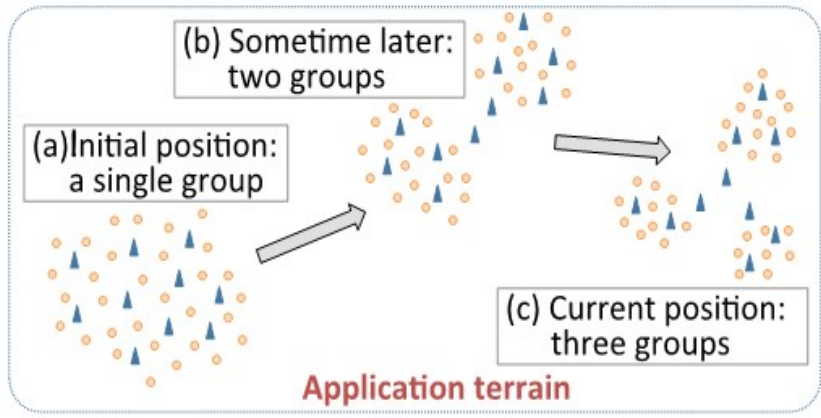

Fig 3.3 AMMNET Model

Clients don't seem to be needed to understand their locations, and solely ought to sporadically probe beacon messages. Once mesh nodes receive the beacon messages, they'll notice the clients inside its transmission vary. With this capability, mesh nodes will ceaselessly monitor the quality pattern of the clients, and move with them to supply them seamless property.

A few assumptions are created in our design. A twodimensional mobile piece of ground, wherever there's no obstacle within the target field. Mesh nodes will exchange data, like their locations and therefore the list of detected clients, with their neighboring mesh nodes. The radio vary of every node isn't an ideal circle in Associate in Nursing application domain with obstacles. This issue could have an effect on the accuracy of the sensing mechanism and, to a minor degree, the coverage. However, this doesn't have an effect on the overall relevancy of the planned techniques for AMMNETs. For simplicity, we tend to assume that the radio vary of each mesh nodes and purchasers may be a good sphere.

\section{ODD-EVEN TURN XY ROUTING ALGORITHM Input:}

Client Node Address CNA, Router Address RA, CoverArea CA, MessagePacket MP, Even column EC, OC Odd Column, East North Direction EN, South West Direction SW

Output: Message Send without Failed MP

For $\mathrm{i}=\mathrm{Max}(\mathrm{CNA}), \mathrm{i}>=\mathrm{RA}, \mathrm{i}++$

Set interval MS to CAN

If RA='True' then Intra-group MS

Switch (RA='True')

Case Intra-group

if Search CAN to RA then

MS send to Intra-group CAN;

If Search CAN neighbors then

Switch Intergroup mode

Else

Random Selection RA to CA

End if

End if

Switch (RA='False')

Case Intergroup Bridge

If $(\mathrm{EN} !=\mathrm{EC} \& \& \mathrm{NW} !=\mathrm{OC}) \& \&$ IF $(\mathrm{ES} !=\mathrm{EC} \& \& \mathrm{SW} !=\mathrm{OC})$

MP forwarded to CAN

Retrieve RA locations

Until $\mathrm{CA}==\mathrm{RA}$ 
End

Else

Message "Deadlock Occur Select Different Sources

Node and destination"

End if End if

End switch

End for

Return MP

\section{ADVANTAGES}

AMMNET victimisation the cluster quality model, AMMNET will really support a a lot of general quality model, wherever clients would possibly move severally. In associate degree extreme case, every shopper will be thought of as a bunch that contains just one user, and therefore the style of AMMNET will still be applied to support property for those freelance clients. To support such a dynamically dynamic network topology, mobile mesh nodes will be classified into the subsequent varieties in line with their current roles during this network:

Intragroup routers. A mesh node is associate degree intramural router if it detects a minimum of one shopper inside its radio vary and is accountable of watching the movement of shoppers in its vary. intramural routers that monitor a similar cluster of shoppers will communicate with one another via multi-hop routing. Intergroup routers. A mesh node is associate degree intergroup router, i.e., sq. nodes in Fig. 4, if it plays the role of a relay node serving to to interconnect totally different teams. for every cluster, we tend to designate a minimum of one intergroup router which will communicate with any intramural routers of that cluster via multi-hop forwarding because the bridge router.

\section{E) DELAY TOLERANT XY ALGORITHM}

AMMNET may be a normal wireless mesh network stationary mesh odes offer routing and relay capabilities. They type a mesh-like wireless network that enables mobile mesh shoppers communicate with one another through multi-hop commutations. Such a network is climbable, flexible, and low in maintenance price. Once a mesh node fails, it will merely get replaced by a replacement one; and therefore the mesh network can acknowledge the new-mesh node and mechanically reconfigure itself. The planned AMMNET has the subsequent extra advantage. The quality of the mesh shoppers is confined to the mounted space serviceable by a typical wireless mesh network thanks to the stationary mesh nodes.

In distinction, associate degree AMMNET may be a wireless mesh network with autonomous mobile mesh nodes. A additionally to the quality routing and relay practicality, these mobile mesh nodes move with their mesh shoppers, and have the intelligence to dynamically adapt the configuration to supply best service. especially, associate degree AMMNET tries to stop network partitioning to confirm property for all its users. This property makes AMMNET a extremely strong painter. The topology adaptation of associate degree AMMNET is illustrated in Fig. 3.4:
- Fig. 3.4a: The mesh clients initially concentrate in one group. All the mesh nodes position themselves within the same proximity to support communications inside the group.

$\triangle$ Stationary mesh node Mobile client

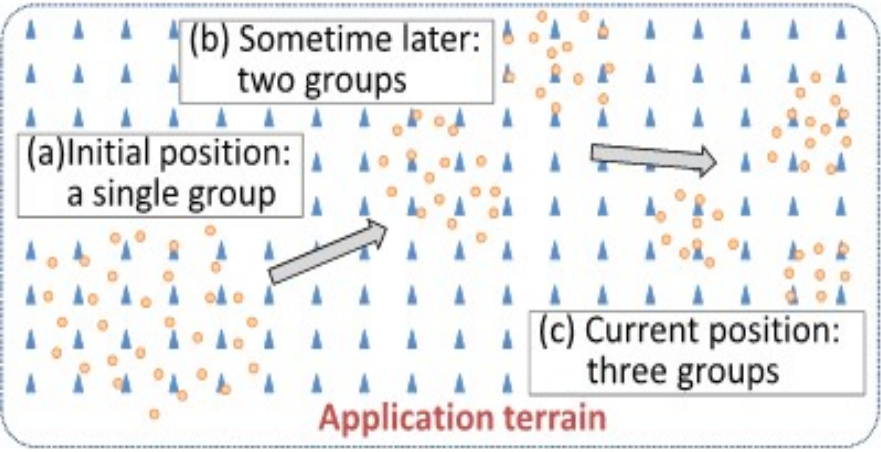

Fig 3.4 AMMNET Fixed grid-based square topology

- Fig. 3.4b: The mesh clients move northwards and split into two groups. The mobile mesh nodes, in this case, reorganize themselves into a new topology not only to facilitate intragroup communications, but also to support intergroup communications effectively preventing a network partition.

- Fig. 3.4c: The same mesh clients now move southeast and form three groups. The mobile mesh nodes adapt their topology accordingly to archive full connectivity for all the mesh clients

\section{V. EXPERIMENTAL RESULTS}

\section{A. XY ALGORITHM}

In $\mathrm{XY}$ routing the addresses of the routers are their xycoordinates. One of the advantages of XY routing is that it never runs into deadlock or livelock [8]. Figure II. XY routing from router $\mathrm{A}$ to router $\mathrm{B}$ in $4 \times 4$ NOC However despite the advantages of simplified calculation and removal of deadlock or livelock problem, there are some problems in the traditional $\mathrm{XY}$ routing.

Table 4.1 describes a XY Routing table analysis. In this table contains GCD routing path, different between node cost and common GCD select given XY routing process details are show.

Table 4.1 XY Routing Table

\begin{tabular}{|c|c|c|c|}
\hline S.NO & Routing Path & $\begin{array}{c}\text { COST } \\
\text { (Assume) }\end{array}$ & $\begin{array}{c}\text { GCD } \\
\{7\}\end{array}$ \\
\hline 1 & $\{1,1\},\{1,2\},\{1,3\},\{1,4\},\{1,5\}$ & 28 & 4 \\
\hline 2 & $\{2,1\}\{2,2\},\{2.3\},\{2,4\},\{2,5\}$ & 35 & 5 \\
\hline 3 & $\{3,1\}\{3,2\}\{3,3\},\{3,4\},\{3,5\}$ & 42 & 6 \\
\hline 4 & $\{4,1\}\{4,2\}\{4,3\},\{4,4\},\{4,5\}$ & 21 & 3 \\
\hline 5 & $\{5,1\}\{5,2\},\{5,3\},\{5,4\},\{5,5\}$ & 14 & 2 \\
\hline
\end{tabular}


Table 4.1.1 XY Results

\section{XY Routing Results}

\section{Normal Routing:}

$4 \rightarrow 5 \rightarrow 6 \rightarrow 3 \rightarrow 2$

XY Routing:

Path Select $2 \rightarrow 3 \rightarrow 4 \rightarrow 5 \rightarrow 6$

The above table 4.1.1 describes a XY Routing process results. The normal path consider 4,5,6,3 and 2 select routing algorithm and another running XY algorithm select the path is $2,3,4,5$ and 6 , because do not occurring deadlock and verified.

\section{B) ADAPTIVE XY ROUTING ALGORITHM}

Adaptive $X Y$ routing works is deterministic or adaptive mode depending on the state of the network. Algorithm works in deterministic mode when the network is not or only slightly congested. When network becomes blocked, the algorithm switches to the adaptive mode and starts to search routes that are not congested. Pseudo adaptive XY routing works on mesh network which consists of routers, wires and IP-blocks. Every router has five bidirectional ports: north, south, east, west and local.

Table 4.2 describes a Adaptive XY Routing table analysis. In this table contains GCD routing path, different between node cost and common GCD select given Adaptive $\mathrm{XY}$ routing process details are show.

Table 4.2 Adaptive XY Routing Table

\begin{tabular}{|c|c|c|c|}
\hline $\begin{array}{c}\text { S.N } \\
\text { O }\end{array}$ & Routing Path & $\begin{array}{c}\text { COST } \\
\text { (Assume) }\end{array}$ & $\begin{array}{l}\text { Commo } \\
\text { n GCD }\end{array}$ \\
\hline 1 & $\begin{array}{l}\{1,1\},\{1,2\},\{1,3\},\{1,4\},\{ \\
1,5\}\end{array}$ & $\{2,4,8,12,10\}$ & $\begin{array}{c}2 \\
{[\{1,1\}-} \\
2]\end{array}$ \\
\hline 2 & $\begin{array}{l}\{2,1\} \\
\{2,2\},\{2.3\},\{2,4\},\{2,5\}\end{array}$ & $\begin{array}{l}\{15,9,15,18,21 \\
\}\end{array}$ & $\begin{array}{c}3 \\
{[\{2,2\}-} \\
9]\end{array}$ \\
\hline 3 & $\begin{array}{l}\{3,1\}\{3,2\} \\
\{3,3\},\{3,4\},\{3,5\}\end{array}$ & $\begin{array}{l}\{21,14,7,28,35 \\
\}\end{array}$ & $\begin{array}{c}7 \\
{[\{3,3\}-} \\
7]\end{array}$ \\
\hline 4 & $\begin{array}{l}\{4,1\}\{4,2\} \\
\{4,3\},\{4,4\},\{4,5\}\end{array}$ & $\begin{array}{l}\{12,28,20,4,40 \\
\}\end{array}$ & $\begin{array}{l}4[\{4,4\}- \\
4]\end{array}$ \\
\hline 5 & $\begin{array}{l}\{5,1\} \\
\{5,2\},\{5,3\},\{5,4\},\{5,5\}\end{array}$ & $\begin{array}{l}\{18,36,30,24,1 \\
2\}\end{array}$ & $\begin{array}{c}6[\{5,5\}- \\
12]\end{array}$ \\
\hline
\end{tabular}

\section{$\underline{\text { Adaptive Routing XY Routing Results }}$}

\section{XY Routing:}

Path Select $2 \rightarrow 3 \rightarrow 4 \rightarrow 5 \rightarrow 6$ routing algorithm and another running adaptive $\mathrm{XY}$ algorithm select the path is 2,3,4,6 and 7 , because do not occurring deadlock and verified

\section{C) Surrounding XY Routing}

Surrounding $\mathrm{XY}$ routing $(\mathrm{S}-\mathrm{XY})$ has three different routing modes. N-XY (Normal XY) mode works just like the basic $\mathrm{XY}$ routing. It routes packets first along $\mathrm{X}$-axis and then along $\mathrm{y}$-axis. Routing stays on NXY mode as long as network is not blocked and routing does not meet inactive routers. SH-XY (Surround horizontal XY) mode is used when the router's left or right neighbor is deactivated.

Table 4.3 describes a Surrounding XY Routing table analysis. In this table contains GCD routing path, different between node cost and common GCD select given Surrounding XY routing process details are show.

Table 4.3 Surrounding XY Routing

\begin{tabular}{|c|c|c|c|c|}
\hline $\begin{array}{c}\text { S.N } \\
\mathbf{O}\end{array}$ & Routing Path & $\begin{array}{c}\text { COST } \\
\text { (Assume) }\end{array}$ & $\begin{array}{l}\text { Com } \\
\text { mon } \\
\text { GCD }\end{array}$ & $\begin{array}{c}\text { Surroun } \\
\text { ding } \\
\text { GCD } \\
\{2\}\end{array}$ \\
\hline 1 & $\begin{array}{l}\{1,1\},\{1,2\},\{1,3\},\{ \\
1,4\},\{1,5\}\end{array}$ & $\begin{array}{c}\{2,4,8,12,1 \\
0\}\end{array}$ & $\begin{array}{l}2 \\
{[\{1,1\}} \\
-2]\end{array}$ & 1 \\
\hline 2 & $\begin{array}{l}\{2,1\} \\
\{2,2\},\{2.3\},\{2,4\},\{ \\
2,5\}\end{array}$ & $\begin{array}{l}\{12,9,15,1 \\
8,21\}\end{array}$ & $\begin{array}{l}3 \\
{[\{2,2\}} \\
\mathbf{- 9}]\end{array}$ & 4 \\
\hline 3 & $\begin{array}{l}\{3,1\}\{3,2\} \\
\{3,3\},\{3,4\},\{3,5\}\end{array}$ & $\begin{array}{l}\{21,14,7,2 \\
8,35\}\end{array}$ & $\begin{array}{l}7 \\
{[\{3,3\}} \\
-7]\end{array}$ & 3 \\
\hline 4 & $\begin{array}{l}\{4,1\}\{4,2\} \\
\{4,3\},\{4,4\},\{4,5\}\end{array}$ & $\begin{array}{c}\{12,28,20, \\
4,40\}\end{array}$ & $\begin{array}{l}4 \\
{[\{4,4\}} \\
-4] \\
\end{array}$ & 2 \\
\hline 5 & $\begin{array}{l}\{5,1\} \\
\{5,2\},\{5,3\},\{5,4\},\{ \\
5,5\}\end{array}$ & $\begin{array}{c}\{60,48,36, \\
24,12\}\end{array}$ & $\begin{array}{l}6 \\
{[\{5,5\}} \\
\mathbf{- 1 2}]\end{array}$ & 6 \\
\hline
\end{tabular}

\section{Surrounding XY- XY Routing Results}

Deadlock Avoid Routing Path

\section{XY Routing}

Path Select $2 \rightarrow 3 \rightarrow 4 \rightarrow 5 \rightarrow 6$

Adaptive XY Routing

Path Select $2 \rightarrow 3 \rightarrow 4 \rightarrow 6 \rightarrow 7$

Surrounding XY algorithm

Path Select $1 \rightarrow 2 \rightarrow 3 \rightarrow 4 \rightarrow 6$

The above table 4.1.3 describes a surrounding XY Routing process results. The normal path consider 2,3,4,6,and7 select routing algorithm and another running surrounding $\mathrm{XY}$ algorithm select the path is $1,2,3,4$,and 6 because do not occurring deadlock and verified 


\section{ODD EVEN IMPLEMENTATION}

Mesh Node Communication (5X5)

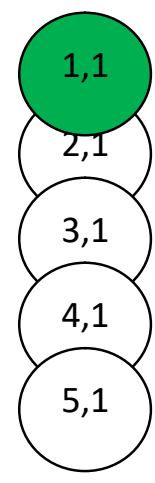

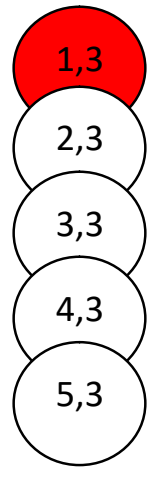

allowed to take an SW turn at any nodes located in an odd column.

[Sources Node $\rightarrow$ Event Column $\rightarrow$ Packet Not send Direction E-S]

[Sources Node $\rightarrow$ Odd Column $\rightarrow$ Packet Not send Direction S-W]

Delay ODD-Even Turn Model Results

Source $\rightarrow \mathbf{1 , 1} \rightarrow$ Path[ [2,1], [3,1], [4,1], [5,1]

$[5,2][5,3][5,4] \rightarrow[5.5] \rightarrow$

Destination

[Communication Vertical to Horizontal

Mesh Node Communication]

Delay XY ODD Even Turn Model (non-

adaptive routing algorithm is deterministic)

Fig 4.1 ODD-EVEN Mesh Network

\section{ODD $\rightarrow$ Path do not Send Packet:}

Rule 1. Any packet is not allowed to take an EN turn at any nodes located in an even column, and it is not allowed to take an NW turn at any nodes located in an odd column.

[ Sources Node $\rightarrow$ Event Column $\rightarrow$ Packet Not send Direction E-N]

[ Sources Node $\rightarrow$ OddColumn $\rightarrow$ Packet Not send Direction N-W]

Rule 2. Any packet is not allowed to take an ES turn at any nodes located in an even column, and it is not allowed to take an SW turn at any nodes located in an odd column.

[ Sources Node $\rightarrow$ Event Column $\rightarrow$ Packet Not send Direction E-S] Direction S-W]

[ Sources Node $\rightarrow$ OddColumn $\rightarrow$ Packet Not send

\section{F: DELAY ODD EVEN IMPLEMENTATION}

\begin{tabular}{|c|} 
ODD-Even Turn Model Results \\
Source $\rightarrow \mathbf{1 , 1} \rightarrow$ Path[ $[1,2],[1,3],[1,4],[1,5],[2,5]$ \\
$[3,5][4,5]] \rightarrow[5.5] \rightarrow$ Destination \\
[Communication Horizontal to Vertical Mesh \\
Node Communication] \\
XY ODD Even Turn Model (non-adaptive routing \\
algorithm is deterministic)
\end{tabular}

Rule 1. Any packet is not allowed to take an EN turn at any nodes located in an even column, and it is not allowed to take an NW turn at any nodes located in an odd column.

[Sources Node $\rightarrow$ Event Column $\rightarrow$ Packet Not send Direction E-N]

[Sources Node $\rightarrow$ OddColumn $\rightarrow$ Packet Not send Direction N-W]

Rule 2. Any packet is not allowed to take an ES turn at any nodes located in an even column, and it is not

\section{Delay ODD-Even Turn Model Results}

Source $\rightarrow \mathbf{1 , 1} \rightarrow$ Path[ [2,1], [3,1], [4,1], [5,1] [5,2] [5,3] $[5,4] \rightarrow[5.5] \rightarrow$

\section{Destination}

[Communication Vertical to Horizontal Mesh Node Communication]

Delay XY ODD Even Turn Model (non-adaptive routing algorithm is deterministic)

Table 4.4 COMPARATIVE STUDY

\begin{tabular}{|c|c|c|c|c|c|}
\hline Metrics & $\begin{array}{c}\text { XY } \\
\text { Routi } \\
\text { ng }\end{array}$ & $\begin{array}{c}\text { Adaptive XY } \\
\text { Routing }\end{array}$ & $\begin{array}{c}\text { Surroun } \\
\text { ding XY } \\
\text { Routing }\end{array}$ & $\begin{array}{c}\text { XY } \\
\text { OD } \\
\text { D- } \\
\text { Eve } \\
\text { n } \\
\text { Mo } \\
\text { del }\end{array}$ & $\begin{array}{c}\text { Del } \\
\text { ay } \\
\text { XY } \\
\text { OD } \\
\text { Dnd } \\
\text { Eve } \\
\text { n } \\
\text { Mo } \\
\text { del }\end{array}$ \\
\hline $\begin{array}{c}\text { Throug } \\
\text { hput } \\
\text { (ms) }\end{array}$ & 0.33 & 0.45 & 0.56 & 0.56 & 0.58 \\
\hline $\begin{array}{c}\text { Averag } \\
\text { e } \\
\text { latency } \\
\text { (\%) }\end{array}$ & 65.3 & 62.3 & 58.4 & 46.3 & 45.3 \\
\hline $\begin{array}{c}\text { Averag } \\
\text { e extra } \\
\text { delay }\end{array}$ & 63.4 & 58.3 & 48.4 & 39.3 & 30.3 \\
\hline $\begin{array}{c}\text { Packet } \\
\text { lost } \\
\text { (count) }\end{array}$ & 90 & 92 & 93 & 100 & 100 \\
\hline $\begin{array}{c}\text { Networ } \\
\text { k } \\
\text { through } \\
\text { put }\end{array}$ & 85.22 & 88.33 & 91.56 & 93.3 & 95.3 \\
\hline
\end{tabular}


From the above findings,

a) Delay XY Odd and Even model has more throughput as compared to XY Routing which is very low.

b) Delay XY Odd and Even model has very low latency as compared to XY Routing.

c) Average extra delay is dramatically reduced in Delay XY Odd and Even model.

d) Network throughput is better in Delay XY Odd and Even model.

\section{CONCLUSION}

This thesis describe reduces the network controller' time in setting routing path data. The bi-connectivity check reduces the time in graph data analysis. The frequent failure nodes are often simply known then potency of network maintenance work is inflated. the top users got to produce nodes and links alone. The applying itself creates the trail and bi-connectivity medical work. In XY routing, we tend to establish the communication between nodes with the data provided within the routing table. If the ordered node is found to be unsuccessful, then the communication wouldn't be lost. If a node features a path means that there exists associate alternate path additionally, so the communication is continued with the given alternate path. This leads to victorious packet transmission for XY routing Model.

In this introduced a mobile infrastructure referred to as AMMNET (XY ODD Even Model). in contrast to typical wireless networks that suffer network partitions once the user teams move apart, the wireless networks mesh routers of associate AMMNET track the users and dynamically adapt the constellation to seamlessly support each their intra-group and inter-group communications. Since this mobile infrastructure follows the users, full property are often achieved while not the necessity and high value of providing network coverage for the complete application parcel of land in the least time as in ancient stationary infrastructure. The thesis describe XY Routing rule a separate node looking logic is given to any or all the neighbor nodes of the disappeared node.

The past communication path is unbroken in memory of all intermediate nodes for given fundamental measure so throughout ordered communication, the previous partial path data is used. In comparative analysis examined XY Routing ODD-Even flip Model for wireless networks, minimizing routing ways, and output, average latency, average additional delay, efficiency, and packet lost and network output.

\section{FUTURE ENHANCEMENTS}

The thesis provides a best assistance in wireless client tracking system management. The following options can be added in future.

- The creation of random links between the set of nodes.

- The routing success ratio can be tracked.

- The routing log can be maintained.
The new system becomes useful if the above enhancements are made in future. The new system is designed such that those enhancements can be integrated with current $\mathrm{XY}$ routing model easily with less integration work.

In future researches, using the deep tree topology find the exact locations of the wireless node. AMMNET that uses locations of the bridge routers to approximate the distribution of the mobile client groups in the application environment and constructs the deep tree; When there are not enough available routers to provide full connectivity for all the clients, this scheme favors user groups-deep tree nodes with a higher density of clients.

In future, implement the backtracking using greedy algorithm to find the disappearing nodes. Backtracking implementation consists in keeping track, at each occurrence of finding deadlock nodes, of all the possible choices that have not yet been tried. This is possible that the dynamic AMMNET framework could support even a larger number of users if they are partitioned into only a fewer number of groups.

\section{REFERENCES}

[1] E. Dahlman, S. Parkvall, and J. Sko * ld, 4G LTE/LTEAdvanced for Mobile Broadband. Academic, 2015.

[2] L. Nuaymi, WiMAX: Technology for Broadband Wireless Access. John Wiley \& Sons, 2017.

[3] K. Fall, "A Delay-Tolerant Network Architecture for Challenged Internets," Proc. ACM Special Interest Group on Data Comm., 2013.

[4] A. Petkova, K.A. Hua, and S. Koompairojn, "Processing Approximate Rank Queries in a Wireless Mobile Sensor Environment," Proc. 11th Int'l Conf. Mobile Data Management (MDM), 2013.

[5] "Quadrocopter LLC," http://quadrocopter.us/, 2013.

[6] R. Roy, Handbook of Mobility Models and Mobile Ad Hoc Networks. Springer, 2011.

[7] Y.-C. Chen, E. Rosensweig, J. Kurose, and D. Towsley, "Group Detection in Mobility Traces," Proc. Sixth Int'l Wireless Comm. And Mobile Computing Conf. (IWCMC '10), 2010.

[8] T. Camp, J. Boleng, and V. Davies, "A Survey of Mobility Models for Ad Hoc Network Research," Wireless Comm. and Mobile Computing, vol. 2, no. 5, pp. 483-502, 2012.

[9] X. Hong, M. Gerla, G. Pei, and C. Chiang, "A Group Mobility Model for Ad Hoc Wireless Networks," Proc. Second ACM Int'l Workshop Modeling, Analysis and Simulation of Wireless and Mobile Systems (MSWiM '15), 2015.

[10] K. Blakely and B. Lowekamp, "A Structured Group Mobility Model for the Simulation of Mobile Ad Hoc Networks," Proc. Second Int'1 Workshop Mobility Management \& Wireless Access Protocols (MobiWac), 2014.

[11] R.V. Boppana and S. Chalasani, ${ }^{a}$ A Comparison of Adaptive Wormhole Routing Algorithms,Proc. Int'l Symp. ComputerArchitecture,pp. 351-360, May 2013.

[12] C.J. Glass and L.M. Ni, aximally Fully Adaptive Routing in 2D Meshes,Proc. 1992 Int'l Conf. Parallel Processing,pp. 101-104,2015.

[13] I.F. Akyildiz, X. Wang, and W. Wang, "Wireless Mesh Networks: A Survey," Computer Networks, vol. 47, no. 4, pp. 445-487, 2015. 
[14] R. Draves, J. Padhye, and B. Zill, "Comparison of Routing Metrics for Static Multi-Hop Wireless Networks," Proc. ACM SIGCOMM, 2014.

[15] R. Draves, J. Padhye, and B. Zill, "Routing in Multi-Radio, Multi- Hop Wireless Mesh Networks," Proc. ACM MobiCom, 2014.
[16] A. Raniwala and T.-C. Chiueh, "Architecture and Algorithms for an IEEE 802.11-Based Multi-Channel Wireless Mesh Network," Proc. IEEE INFOCOM, 2015.

[17] M. Alicherry, R. Bhatia, and L.E. Li, "Joint Channel Assignment and Routing for Throughput Optimization in Multi-Radio Wire-less Mesh Networks," Proc. ACM MobiCom, 2015. 